\section{Use of Micro-Computed Tomography for Bone Evaluation in Dentistry}

Milena Suemi Irie ${ }^{1}$, Gustavo Davi Rabelo ${ }^{1}$, Rubens Spin-Neto², Paula Dechichi $^{3}$, Juliana Simeão Borges ${ }^{1}$, Priscilla Barbosa Ferreira Soares ${ }^{1}$
ISSN 0103-6440

'Department of Periodontology and Implantology, UFU Universidade Federal de Uberlândia, Uberlândia, MG, Brazil

${ }^{2}$ Department of Dentistry and Oral Health, Aarhus University, Aarhus, Denmark 3nstitute of Biomedical Sciences, UFU - Universidade Federal de Uberlândia, Uberlândia, MG, Brazil

Correspondence: Priscilla Barbosa Ferreira Soares, Av. Pará, 1720, Bloco 4L, Anexo A, 38400-902, Campus Umuarama, Uberlândia, MG, Brasil. Tel: +55-34-3225-8119. e-mail: pbfsoares@yahoo.com.br

Key-Words: bone, microcomputed tomography, resolution, segmentation, texture analysis.

\section{Introduction}

Micro-computed tomography $(\mu \mathrm{CT})$ follows the basic principles of medical computed tomography in which the sample is placed in the path of an $\mathrm{x}$-ray beam forming a projection image on the scintillator or other $x$-raysensitive detector array (1). The sample is rotated and imaged at a large number of angles, and the sequence of projection images is "back-projected" to reconstruct the $x$-ray absorption at each point within the scanned volume $(1,2)$. The $\mu \mathrm{CT}$ is currently used to evaluate bone morphometric characteristics as the complimentary alternative of conventional histological analysis (2), when considering a tridimensional volume of the sample over two-dimensional histological sections. Using $\mu \mathrm{CT}$ allows a more representative analysis in the whole sample extension, being the most advantage compared to histology analysis, although, it should be emphasized that histology remains as the most indicated method to evaluate cells, proteins and composition.

The $\mu \mathrm{CT}$ as non-destructive method provides 3D information without destroying the sample, allowing its inner reconstruction by radiographic image sections. The same sample can later be prepared for histologic analysis, generating complementary information of the same tissue areas. In addition, using $\mu \mathrm{CT}$ it is possible to measure bone quality based on other parameters such as mineral properties. Also, microarchitecture analyzed in a representative bone volume will assess distinct sites, and not just on the few sections provided in histology methods (3).

$\mu C T$ and HR-pQCT (high-resolution peripheral quantitative computed tomography system) are nowadays the most useful high-resolution imaging of trabecular and cortical bone ultrastructure (4). The $\mu \mathrm{CT}$ equipment's are available for in vivo and in vitro imaging for bone analysis. $\mu \mathrm{CT}$ has been widely used in medicine and dentistry fields to assess the bone quality defined by several structural parameters, making possible to evaluate the repair and regeneration of the bone tissue in animal and human samples (5). A large number of studies have used $\mu \mathrm{CT}$ in order to identify alterations and changes due to systemic diseases and conditions (e.g. osteoporosis, osteoarthritis, hypercalcemia, diabetes, etc) and also metabolic diseases and their respective treatment (e.g. anti-resorptive treatment, corticoids, etc) (6). High correlations and excellent agreement between conventional histomorphometry and $\mu \mathrm{CT}$ data have been demonstrated (7).

At the largest length scales, two types of bone structure are evidenced: trabecular and cortical bone. Trabecular or cancellous bone micro-architecture shows a porous network with small filaments inter-woven which results in higher porosity (80\%-85\%) comparing to cortical area (2-5\%) $(7,8)$. Trabecular bone can be 10 times thinner than the 
cortical structure. High resolution images are necessary to analyze trabecular portions, particularly in rodent models, which can exhibit $0.2 \mathrm{~mm}$ of thickness in healthy mice (9). Cortical bone should be evaluated concerning structure by CT methods, and possible alterations on cortex structure are relevant to bone strength. If the cortex is thick, images of low resolution may be sufficient for analysis, however, if the cortices are thin, measurement of the morphological features could be a challenge (10).

Bone quality involves bone mass, structural properties: geometry, macro and micro-architecture, and tissue properties: modulus of elasticity, mineral density, collagen quality, cell and marrow behavior (11). Mechanical and biological behavior of bone tissue play important role in clinical practice, especially for evaluating distinct systemic and local conditions (diseases, therapies or lesions). This property can also contribute to better understanding of bone regenerative procedures, such as: alveolar ridge and maxillary sinus augmentation procedures, bone repair and fracture healing (12), surgical reconstruction and rehabilitation, and implant osseointegration (13). $\mu \mathrm{CT}$ has shown to be the most indicated technique for assessment of the bone mass and morphology in animal models (3). In

addition, particularly in dentistry, this method is extremely useful to the study of human jaw bone tissue associated with different conditions and diseases, also to assess the changes when the bone is evolved by lesions or submitted to surgical procedures (14-16).

Before starting a $\mu \mathrm{CT}$ analysis, it is important to previously define all the crucial parameters to answer the study question. Variables in the analysis should be considered, including: type of the sample (i.e. bone site, size, thickness); sample preparation (not allowing dryness, excessive heating, mimicking the surrounding tissues as muscles); the next step of the assessment (histological processing, mechanical tests); the necessary resolution (different equipment, in vivo or in vitro condition, influence on binarization process), and at last the parameters to be evaluated (e.g. considering separated analysis of cortical and cancellous bone, bone repair, etc). Therefore, the aim of the present study was to review and discuss the equipment, sample preparation, scanning parameters, and analysis methods applied to bone tissue evaluation in dentistry using $\mu \mathrm{CT}$-based methodology.

\section{Review of Literature Before Image Acquisition Sample Preparation}

For bone tissue analyses, the sample preparation and the stabilization of the sample holder inside the $\mu \mathrm{CT}$ unit should be standardized. Some systems have an open platform to fit the sample, so it is important to make sure that the entire sample fit this space and remain inside the field of view during the whole scanning (in $180^{\circ}$ or $360^{\circ}$ rotation). Otherwise, the image has to be reconstructed locally reducing image quality (17). Sample preparation will vary according to the type of specimen, however mostly samples are excised, and then the soft tissue may be removed. Moreover, specimens can be aligned with the vertical or horizontal axis of the unit, in this case the vertical positioning is more adequate. Horizontal placement is compromised with a slightly reduced image quality due to beam-hardening along and perpendicular to the long bone. No relative displacement is desirable during the measurement; if the sample changes the initial positioning during the scan, motion artifacts may appear (3).

The $\mu$ CT scanning of bone tissue can be done by maintaining the sample in liquid or air environment. However, if quantitative measurements of tissue mineral density are required, is recommended to scan in a liquid medium. Changes in medullary density can happen if the bone is dehydrated. Scans can take 10 to 11 hours, to avoid sample dehydration and shrinkage during scanning is recommended to scan samples inside liquid-filled tubes. The operator needs to assure all specimens are scanned in the same type and volume of the medium. Saline, ethanol, and neutral buffered formalin medium have been used for bone storage medium during $\mu C T$ scanning (18). If the specimens are stored in ethanol, they should be rehydrated in saline solution $(0.9 \%)$ by overnight immersion or over a weekend before scanning. Bone samples also can be harvested without chemical fixation and store frozen at $-20^{\circ} \mathrm{C}$ immersed in saline inside a tube or wrapped in saline-soaked gauze (19). The bone samples can also be fixed and stored in the phosphate-buffered saline (PBS). However, attention should be given in cases of long term storage in PBS, because partial change to acetic acid may occur, leading to bone demineralization (20). To avoid air bubbles formation, all these liquids should be inserted using a syringe (3). To maintain normal hydration of bone during scanning, the specimens can be wrapped in paper tissue, inserted into a tube and then, moister the paper with water or saline solution to avoid dehydration during scanning (20). If the sample will follow for histology after $\mu$ CT scan, first it should be fixed (usually in paraformaldehyde), and during the scan it should be kept in a saline or buffered solution. In cases of biomechanical tests and $\mu \mathrm{CT}$ performed in the same sample, for example, the sample should not be fixed and maintained frozen until scan.

\section{Scanning and Quantitative Assessment Image Acquisition and Image Resolution}

Before scan, some parameters should be defined in order to have better quality in the images that will be 
reconstructed. Besides resolution and pixel size, the operator should define the use of filters. Aluminum or copper filters are used in order to reduce beam-hardening artifacts (3). By experience, the most used filter for bone evaluation is the aluminum with $0.5 \mathrm{~mm}$.

Concerning resolution, the pixel size chosen will define the voxel size in the following step, i.e the tridimensional reconstruction. Voxel unit from $\mu \mathrm{CT}$ is denominated as isotropic voxels because it has all three equal dimensions. The resolution chosen for a $\mu \mathrm{CT}$ volume has strong influence on the results of cortical and trabecular bone analysis if the voxel size is not compatible with the structures size (21). This influence is not relevant when thick trabecula is analyzed, such as human sample (100 to $200 \mathrm{~mm}$ ). However, results from small structures (20 to $60 \mathrm{~mm}$ ), like rodent's trabeculae, can be affected by voxel size resolution $(7,22)$. Three-dimensional reconstructions of serial sections led to a new understanding of the branching patterns and remodeling processes of the cortex (2). The relation between voxel size and scanning time should also be considered. Scanning small and highly details structures with low resolution $(>100 \mu \mathrm{m})$ can underestimate bone mineral density and overestimate sample thickness (3). Ideally, the smallest voxel size available should be used in order to obtain the highest amount of detail possible. However, resolution is time dependent, and high-resolution scanning (small voxel size) results on longer acquisition and reconstruction time, and large data sets. If $\mu \mathrm{CT}$ scanning is performed on live animals (i.e. in vivo) for long time, the high dose of radiation might become relevant and threaten the animal's health state (23).

When the ratio between voxel size and sample size decreases, in general, the measurement error is minimized. Thus, the ratio should be lower for more accurate $\mu \mathrm{CT}$ performance (3). Using the $\mu \mathrm{CT}$ evaluation based on nominal voxel sizes of $6,10,15,20$, and $30 \mu \mathrm{m}$ there was a strong effect on the results of several commonly reported trabecular bone parameters in mice vertebral body trabecular bone (23). Since mice trabecular dimension is approximately $30 \mu \mathrm{m}$, scanning with larger voxel sizes can result on results which are poorly accurate. The trabecular bone structure outcomes are significantly affected by $\mu \mathrm{CT}$ scanning voxel size and by the global segmentation method (i.e. thresholding) used to separate bone from non-bone (23). Therefore, it is recommended that high-resolution scans should be used whenever possible to provide the most accurate estimation of trabecular bone microstructure.

\section{Regions of Interest: Cortical and Trabecular Bone}

To determine the region of interest (ROI) of a sample it is important to define the interest landmarks, the contoured region of interest, and the size. The starting point should be known anatomical sites (i.e. landmarks), such as the proximal tibial plateau, the metaphyseal growth plate or another appropriate anatomic site in animal samples. For human samples, distinct characteristics should be considered, meaning differences between maxilla and mandible. According with the study question, the region analyzed in animal models should rely on the criteria: a) will cortical and cancellous bone be analyzed?; b) in studies of bone repair, the created lesion will be performed within the epiphysis or the diaphysis?; and for human studies, the criteria should follow similar questions: a) is it important to analyze cortical and cancellous bone separately?; b) should larger samples be segmented in smaller pieces?; c) is there standardization among the samples?; and just after these definitions, together with the criteria about the scanning phase (resolution, scanning time, sample preparation), the region of interest should be defined. The ROI size should be defined as the distance from the start point until the end of the defined region, set by number of sections to be scanned, based on the defined voxel size (3). The area and volume of the bone to be analyzed will depend on the type of study. To analyze trabecular bone in rats or mice, the metaphyseal region of long bones is mostly located a few millimeters adjacent to the metaphyseal growth plate. If the volume of interest is out of this region, more medullar areas are included reducing the mean value for the ratio bone volume/total volume. Thus, relevant differences between study groups can be masked (24). For the cortices, usually is selected diaphysis region, for the measurements of the cortical area, mean cortical thickness and total bone. For bone repair and regenerative procedures, the area should contain the whole lesion, in order to assess the new bone formation (Fig. 1).

\section{D Reconstruction}

A volume image obtained from $\mu \mathrm{CT}$ consists of a stack of cross sections reconstructed according to the axis of rotation of the unit's gantry. The reconstruction software usually generate voxels that are isotropic, and the voxel dimensions are automatically calculated with the same accuracy as the imaging system's calibration (1). The bonetissue reconstruction allows the identification the specimen section by section. Then it is possible to analyze the whole volume by the delimitation of the region of interest (ROI), including cortical and cancellous bone (Fig. 2). In X-ray microtomography, image information from a voxel with a very small physical size should be detected and the right choice of parameters for noise reduction becomes very important in the reconstruction step.

During reconstruction, the software usually allows to work on histogram, and also, to perform corrections such as: smoothing, ring artifacts reduction and beam-hardening 

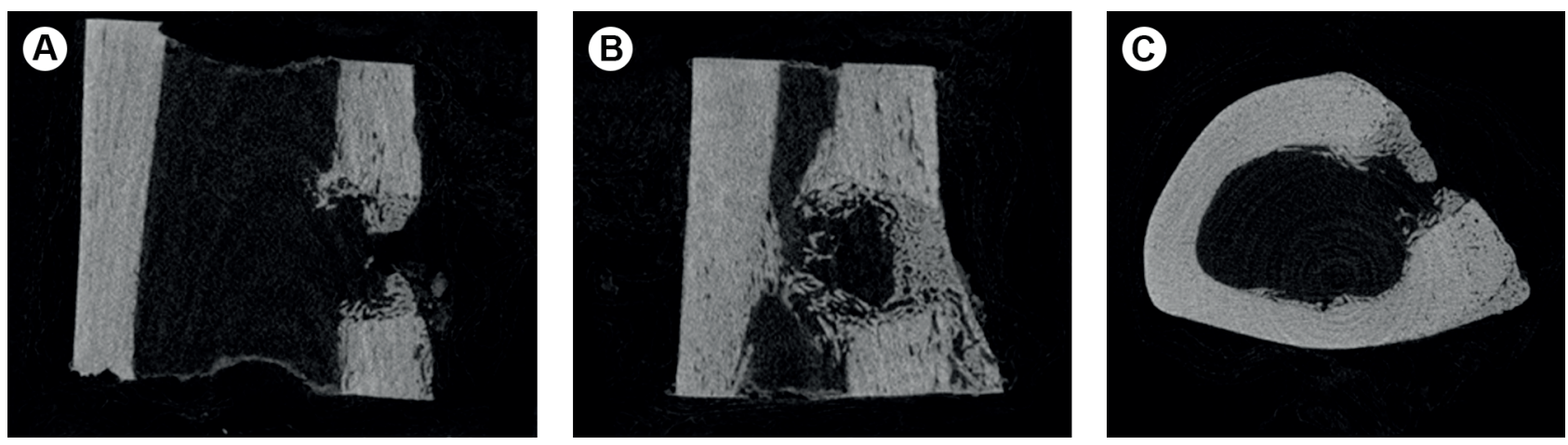

Figure 1. Rat femur with bone repair process evaluated 7 days after euthanization revealing new bone formation in the region of the created lesion using a drill (8 $\mu \mathrm{m}$ pixel size at $90 \mathrm{kV}, 111 \mu \mathrm{A}$ and $720 \mathrm{~ms}$ integration time; Rotation step 0.400 and Filter Al $0.5+\mathrm{Cu} 0.038)$. A: Coronal view. B: Sagittal view. C: Trans axial view.
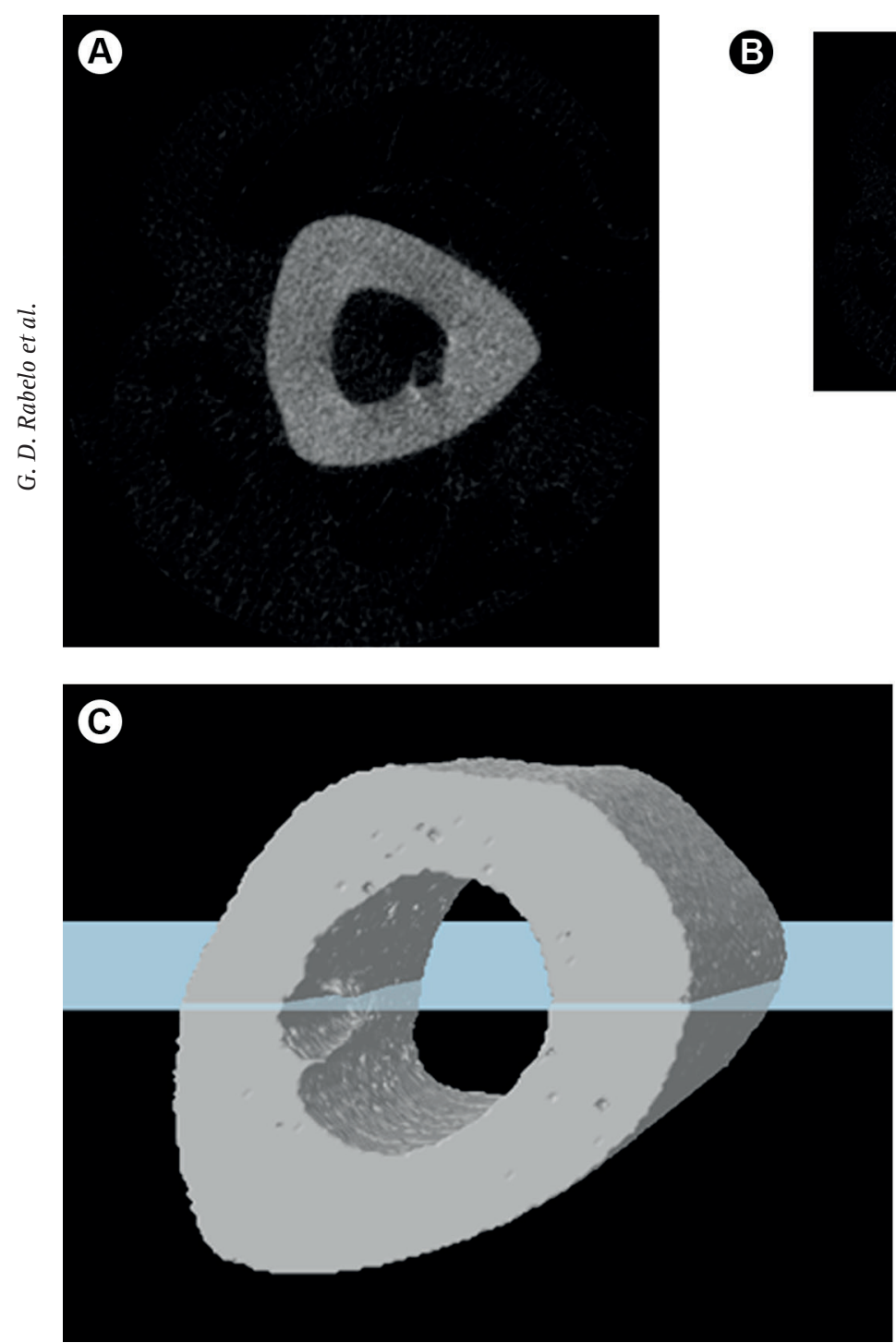

B
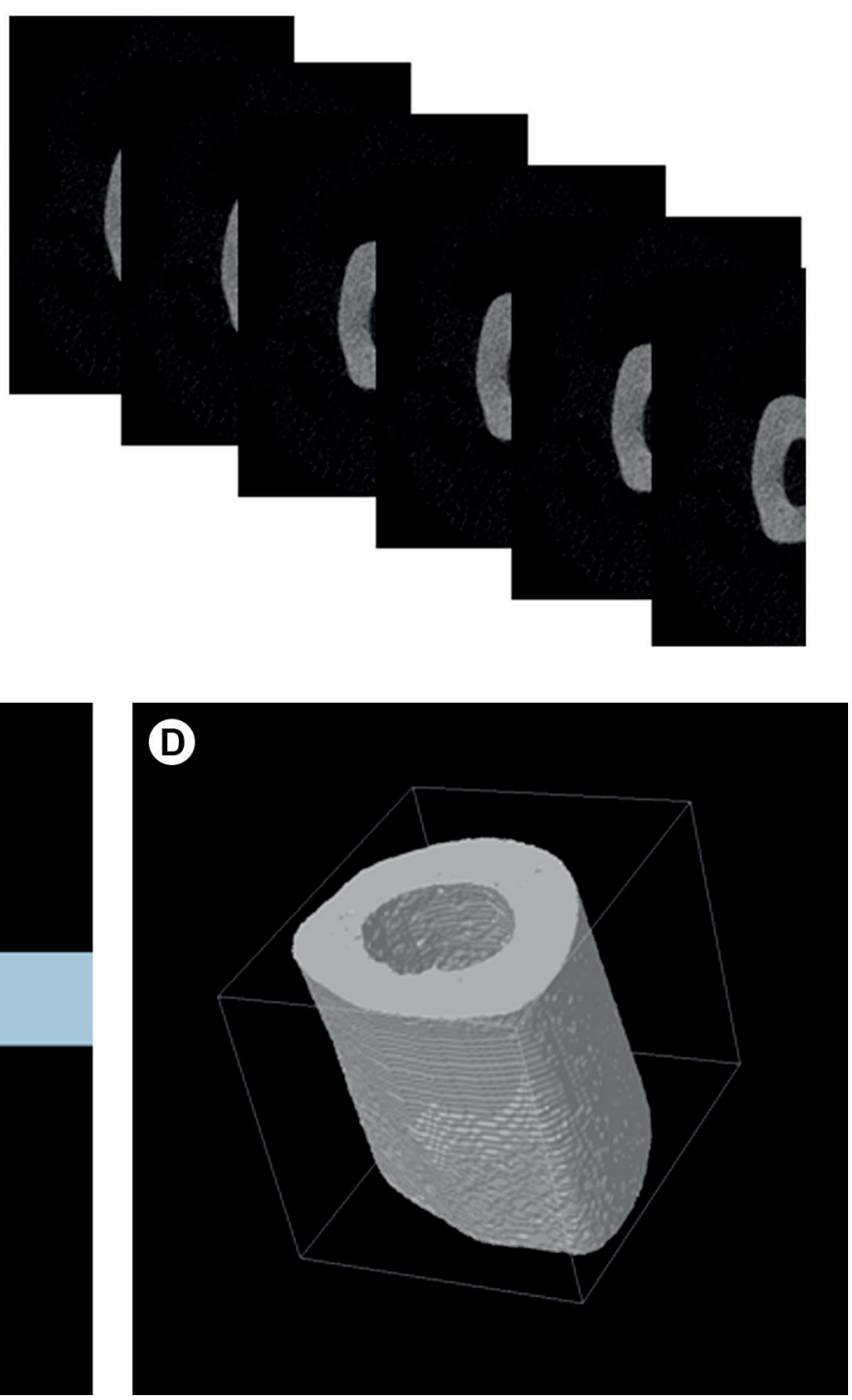

Figure 2. Rat tibiae scanned at the diaphysis in order to evaluate the cortices $(15 \mu \mathrm{m}$ pixel size at $90 \mathrm{kV}, 111 \mu \mathrm{A}$ and $1800 \mathrm{~ms}$ integration time; Rotation step 0.600 and Filter $\mathrm{Al} 0.5+\mathrm{Cu}$ 0.038). A: One single slice in gray-scale image revealing the cortex and the medullar channel in the center. B: Representation of the slices grouped in a seriated organization in order to allow the 3D reconstruction. C: 3D volume of the whole scanned segment of the tibiae (in gray) and the plate (in light blue) that can be used to segment the volume to visualization of the inner structures of the bone. D: Another view of the 3D volume obtained from the serial sections. 
correction. The use of this tools enable to a more accurate 3D volume for analysis and noise reduction, however, they could increase the time of the reconstruction step.

\section{Choosing the More Appropriated Parameters: What} is the Study Question?

The parameters evaluated should answer the major study questions, so the acquired data will provide information for the major deliverables of the project. The use of $\mu \mathrm{CT}$ evaluation should be able to represent the structural organization (microarchitecture) and the bone composition (mineral density defined by X-ray absorption). Using three-dimensional imaging techniques like $\mu \mathrm{CT}$, more complete information of bone microarchitecture became available, and it is already known that the complexity of the microarchitecture will have an important influence on the mechanical competence of bone (25). Bone strength analysis relies on the evaluation of intrinsic (mineral and collagen quality, presence of microcracks) and extrinsic determinants (geometry, bone mass and mineral density, macro, and microarchitecture) (11). The $\mu \mathrm{CT}$ method will provide crucial information on some of these determinants. The identification of changes in these determinants will aid to understand the influence of structure and composition on strength, toughness and stiffness of bone.

In human or animal bone, is possible to assess bone quality features by means of $\mu \mathrm{CT}$ with the use of X-rays. It is necessary to keep in mind what are the best parameters in cortical and cancellous bone that could differentiate two different conditions (i.e disease versus control). In addition, it should be possible to characterize different phases and the evolution of the specific situation in the study, such as in the cases of bone repair evaluation, fracture healing or osseointegration of the biomaterial or implant.

\section{Binary Selection: Choosing the Threshold}

An image is mathematically represented by a matrix of numbers, which contains a numerical value correspondent to the intensity value or pixel depth. Each image matrix element contains a numerical value that corresponds to the image intensity value or pixel depth (26). In $\mu \mathrm{CT}$ imaging, these intensities are often presented within a 8-bit grayscale, in 256 levels of gray, varying from 0 to 255 . When proceeding binary selection, it means the process to convert the X-ray image acquired, and which is present in 256 levels of gray, to only two "colors": one including and the other excluding the evaluation tissue. To perform such step, the definition of a threshold which separates what is and what is not tissue, is rather important. Choosing two thresholds (upper and lower), defining a range of what to be considered as bone, will enable to differentiate the bone from the holes (non-bone material), in order to use the numeral values obtained from each region to make the measurements. The question is whether the automatic segmentation from bone and non-bone parts within the image could compromise the final evaluation. The use of a global threshold refers to the use an automatic, easy and non-time consuming way to separate and differentiate the bone, however, careful should be taken in attention to this automated process. Problems such as noise, resolution limitations, and beamhardening will be critical in the segmentation of the original reconstructed grayscale data sets to separate bone from non-bone. Inappropriate segmentation method will reduce the potential power of $\mu \mathrm{CT}$ and may introduce bias in the architectural measurements (27).

There is no consensus on a threshold that could be used for all studies, and extreme care must be taken in the definition of the best threshold (3). For instance, selecting a global threshold in studies where bone mineralization may not be constant (e.g. during bone repair) or when there are extreme ranges of bone volume fraction among groups, and one unique value is not adequate. In dentistry, using human or animal bone, the threshold should be defined taking into account the type, site, and inherent bone characteristics. It is necessary to distinguish the evaluation of cortical and trabecular bone separately. Also, when the region of interest includes bone repair or healing (i.e. the region of a created lesion for bone repair evaluation, the site of osseointegration of the implant or biomaterial, or the tooth extraction area - Fig. 3), should also be delimited and separated from the other parts included in the field of irradiation. The ability to measure cortical and trabecular bone density and architecture separately with highresolution methods has enabled to better understand the changes that occur with age, diseases, differences between sexes and races, and the effects of drug treatment (28).

\section{Cortical Bone Evaluation}

Evaluation of the cortices should assess initially two important parameters: thickness (Ct.Th) and porosity (Ct.Po). The optimum resolution chosen is that one which allows the identification of the bone channels, including even the smaller ones. To assess cortical porosity in humans it should be known that the bone channels vary according to the site, age and systemic condition. In long bones they have a mean diameter of about $60 \mu \mathrm{m}$ in aged women (29). In animals, for adult rats we found a mean diameter of $7 \mu \mathrm{m}$ and for rabbits around $15 \mu \mathrm{m}$ (unpublished data). The threshold should delimitate the bone matrix separated from the holes to make possible to reach the intracortical porosity by means of calculation of all pixels that do not correspond to bone tissue. By porosity, it is also possible to assess the differentiation between the open and closed porosity. When the open porosity is chosen, it will take 
into account the open channels on the surfaces included in the ROI. On the other hand, the closed porosity will be related just to the intracortical porosity, by means of that only closed pores (channels) will be considered in the analysis. Besides thickness and porosity, the $\mu \mathrm{CT}$ software also allows identifying other characteristics, such as: bone surface area (Ct.BS), which in this case is related to all pixels on the contour of all channels within the region of interest. Additionally, other features, like the percent of bone volume, meaning the inverse counting of pixels compared to the porosity are also possible to obtain. It should be emphasized that the majority of the studies had focused on the trabecular bone for the evaluation of bone fragility; however, the cortex is the major dictator on this characteristic (10). Non-vertebral fractures occur predominantly at cortical sites and most of bone loss occurs by intracortical remodeling, that cavitates the cortex producing porosity, being more important in these cases the assessment of cortex than trabecular bone (10).

\section{Cancellous Bone Evaluation}

The trabeculae or "trabecular" bone is the primary anatomical and functional unit of cancellous bone, with high turnover rates compared with the cortical bone. In the prediction of bone strength, measurements of trabecular density and trabecular microstructure should be combined and performed in combination with cortical evaluation. Trabecular bone morphometry depends on the measurement of the bone volume (BV) and also on the characteristics of the trabeculae including: the trabecular number (Tb.N) calculated as the average number of trabeculae per unit length; the trabecular thickness (Tb.Th) as the mean thickness of trabeculae; and the trabecular separation (Tb.Sp) as the mean distance between trabeculae.
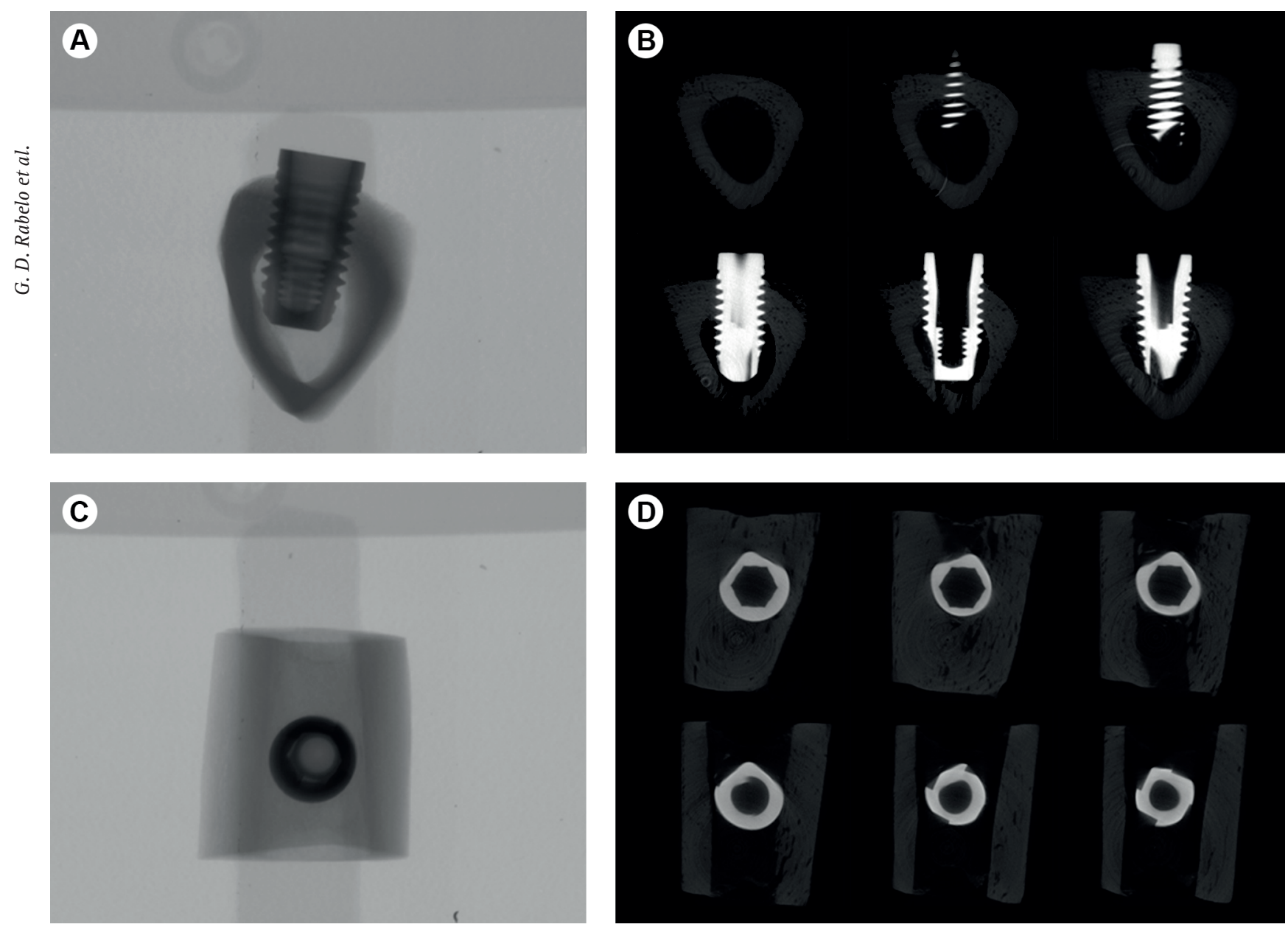

Figure 3. Implant installed in a rabbit tibia $(8.5 \mu \mathrm{m}$ pixel size at $90 \mathrm{kV}, 278 \mu \mathrm{A}$ and $1320 \mathrm{~ms}$ integration time; rotation step 0.400 and Filter Cu $0.1 \mathrm{~mm}$; Reconstruction: Smoothing=2, Smoothing kernel=0 (Asymmetrical boxcar), Ring Artifact Correction=8 and Beam Hardening Correction $(\%)=80)$. A: $\mu$ CT original file in a coronal view of the implant inserted in the tibia. B: Random images representing a sequence of the slices going through the implant, starting from the tibia without the implant on the upper left corner and finishing at the end of the implant in the lower right corner. C: $\mu \mathrm{CT}$ original file in a transaxial view of the implant inserted in the tibia. D: Random images representing a sequence of the slices going through the implant, starting from the superior part of the implant inserted within the cortex on the upper left corner and finishing at the end of the implant in the inferior part, inside the medullary portion in the lower right corner. 
By means of algorithms included in the analysis software, it is also possible to measure the connectivity density (Conn.D), as one measurement that gives information on the trabeculae degree of connectivity and the structure model index (SMI). SMI is an indicator of the trabeculae format, being the ones with the value more close to 0 related to the parallel ones, and a value more close to 3 meaning more cylindrical rods shape of the trabeculae (3). In osteoporosis for example, there is a conversion of plates into rods (6). Besides these parameters, others also could be measured as the bone surface density, but the majority of the studies in the literature focused on the ones previous mentioned.

\section{Additional Analysis and "Unthresholded" Methods}

Additional measurements using $\mu \mathrm{CT}$ are available through developed algorithms in order to assess architecture parameters and features do not associated with the classical analysis. The spatial organization of the cortical and trabecular bone gives additional information how these tissues are structured and the influence on their mechanical properties. The texture analysis enables to reach improvements in bone microarchitectural evaluation from low resolution images (e.g. radiographs, Dual-energy X-ray absorptiometry - DXA) or add a new mathematical way to measure structure parameters, based on the size, shape and distribution of elements within the tissue. Some parameters of the texture analysis are included in the conventional $\mu \mathrm{CT}$ software's, and others can be acquired using plugins and download free software's, like Image J (NIH). This analysis evaluates local variations in gray levels, the degree of complexity, given by numerical values as how a structure fills up the space; and quantifies the emptiness, by an indicator of the degree of gap distribution over a certain surface $(30,31)$. The most used additional analysis on $\mu \mathrm{CT}$ are the degree of anisotropy, fractal dimension, and lacunarity, disposable in the evaluation software, except for the lacunarity.

Emphasizing anisotropy measurement, the degree of anisotropy gives information on the orientation of trabeculae in space. A preferential orientation of the trabecular plates observed along the direction of the strains exerted on bone is related to high anisotropy (6). Concerning the cortex, cortical structure follows a preferential orientation and hierarchical arrangement, inducing strong anisotropy (32).

The Fractal Dimension (FD) is another parameter that may be extracted by $\mu \mathrm{CT}$ analysis. This parameter indicates how much an object occupies its underlying metric space, meaning that is a measure that characterizes how much an object occupies the space that contains it (31). The fractal geometry analysis has been used for describing irregular patterns with self-similarity at different scales, for example, it has been used to characterize the microstructure of porous media within the cortical bone (33). Fractal geometry analysis of cancellous bone identifies architectural features not easily recognized by conventional bone histomorphometric (34). A decrease in fractal dimension was already evidenced when there are alterations of trabecular bone, meaning loss of trabecular connectivity and increase in porosity (35). The complexity of trabecular bone microarchitecture, expressed by the mean value of the fractal dimension, was higher in cases of fracture compared with control cases (36).

Another important parameter is the lacunarity, which measures the gap or lacuna distribution, meaning higher the lacunarity, bigger will be the variation of pixels distribution within an image. High lacunarity means that pixels are grouped in a wide variety of sizes of islands, surrounded by a widely variant emptiness, indicating heterogeneity of spatial pattern or texture (31). Lacunarity plots characterize the spatial organization of an image, including the average size of any structural sub-unit(s) within the image, making them potentially useful in representing the trabecular thinning and perforation of vertebral trabecular bone associated with osteoporosis (37). In dentistry, the lacunarity measurements should be employed attempting to understand the heterogeneity of bone channels network in cortical bone and to evaluate the homogeneity in the trabeculae distribution in cancellous bone. Lacunarity decreases with the increase of regularity of gaps distribution (38). This parameter can be measured in both threshold and non-threshold methods, using free download software's. The use of non-threshold images (original acquisition images), without binarization, could overcome some problems like low resolution, once the measurement works with the distribution of gray-level pixels in the image.

\section{Results of $\mu \mathrm{CT}$ Evaluation: Interpretation and Significance \\ Morphometry and Mineral Density Measurements: Clinical Significance}

Morphometry, an important parameter extracted by $\mu \mathrm{CT}$ analysis, is related to the measurement of bone architecture. Data obtained for analysis of the different conditions of bone integrity, reflects on the biomechanics behavior of this connective tissue. Using $\mu \mathrm{CT}$, the assessment of the spatial organization of bone structure and their mineral composition is possible to identify alterations caused by diseases or physiological adaptation, with important clinical significance.

Data extracted from $\mu \mathrm{CT}$ datasets make possible to explore the bone architecture in its macro and microscale. 
When the bone quality is compromised, alterations in trabecular bone are evidenced. Trabecular bone volume, trabecular thickness and trabecular number are significantly decreased in osteoporosis (39) and in osteoarthritis (40). Bone trabeculae decreases in aging due to constant osteoblastic depression, and this usually leads to a progressive conversion of the trabeculae format from plates into rods (6). In relation to cortical bone, cortical thickness and porosity are the parameters that are directly related to bone strength, so being crucial concerning the clinical significance when analyzing the bone in diseases or distinct conditions. Cortical porosity is increased in several diseases, with increased osteoclast resorption activity. Cortical bone porosity is responsive to disease, therapy, and metabolic alterations, with the pore structure intimately related to biomechanical properties, for example, with the pores becoming increasingly interconnected and convoluted as age progresses (2). This cortical bone loss is one of the major responsible for the reduction in strength. The porosity is increased in diseases like diabetes (41), chronic kidney disease (42), osteoporosis (39), rheumatologic diseases and others, which present high risk of fractures. All these conditions have clinical relevance, and studies conducted $\vec{s}$ in human or animal studies should apply techniques that are able to evaluate the bone quality, such as the $\mu \mathrm{CT}$.

\section{Animal Studies: Bone Repair, Modeling, and} Remodeling

The majority of studies concerning bone repair are concentrated in the application of synthetic bone substitutes, biomimetic molecular agents, physical stimulation, systemic or local conditions known to have deleterious effects on bone, or a combination of them $(43,44)$. The evaluation of the bone repair process in conditions that affects bone turnover and metabolism, may be compromised by external causes and systemic conditions, can be investigated by using $\mu \mathrm{CT}$. Experimental studies in rats revealed alterations in bone healing, for example due to radiotherapy, which affects the bone repair (12). For evaluation of dental implants osseointegration process, the $\mu \mathrm{CT}$ associated with animal model also is commonly used, and allows the assessment of the new bone formation process around the implant. Nevertheless, some of these studies were focused on histomorphometric findings and have some limitations, one of them related to the $2 \mathrm{D}$ evaluation of a few slides. By using $\mu \mathrm{CT}$, the advantage could be the 3D evaluation by assessing the whole bone repair process, including the whole microstructure of the new bone formation.

The bone healing cascade follows a process of: an early phase that enables temporarily fracture stabilization and further endochondral bone formation, characterized by the recruitment of mesenchymal cells and successive chondrogenesis resulting in a soft callus formation. The second stage is distinguished by deposition of the collagen and subsequent mineralization resulting in a woven bone formation and the last stage is characterized by the bone remodeling which restores the original bone structure and strength (44). Irrespective of the study question, using $\mu \mathrm{CT}$ by means of the whole 3D volume representative of the bone repair region, it is possible to achieve answers which provide insights into how the bone neoformation and healing took place (Fig. 4).

In the evaluation of new bone formation, when using $\mu \mathrm{CT}$ analysis, the bone volume (BV) is the most important assessed parameter. It means the new bone amount within the region of interest, represented by the lesion filled with bone, or the callus on fracture healing, or even the new bone surrounding implants. The same parameters for the trabecular bone can be evaluated. The characterization of the new bone trabeculae formation, and in the late phase, new formed cortices also can be evaluated by the parameters described previously (Fig. 4). Additionally, the parameters related to bone composition can be measured and differentiate the new bone, like bone mineral density (BMD) measure as the volumetric density of calcium hydroxyapatite (CaHA) in terms of g.cm-3. By $\mu \mathrm{CT}$ of fracture healing, using different ranges of voxel brightness (thresholds) to identify mineralized tissue of different densities, with values in a range for less dense new bone and calcified cartilage, another range for very dense cortical bone and other range for non-mineralized tissue (45).

\section{Final Recommendations}

The use of $\mu \mathrm{CT}$ for bone tissue evaluation should be extensive and thorough, taking into consideration the maximum possible tissue extension through the whole tridimensional reconstruction of the sample.

The bone samples should be prepared and maintained in an adequate medium before and during the scanning. If histological processing will follow the scan, the correct fixation of the material should be made (with alcohol or paraformaldehyde, according to the next steps of the sample processing). Although, if is the case to perform mechanical tests, the storage should take place in adequate frozen conditions.

Small and standardized bone samples will provide better scanning conditions, with influence on the final results regarding the possibility to scan with better resolution in this condition.

With regards to image acquisition, the resolution should be able to give accurate results concerning the structures to be evaluated. In cortical bone, the better resolution should allow the identification and measurement of the bone 
channels, even the smaller ones. In cases that is necessary to assess the osteocytes lacunae, high-resolution around $1 \mu \mathrm{m}$ can be defined, but the longer scan time and a small analyzed area should be considered.

Trabecular bone should be evaluated in order to allow the correct identification of the trabeculae, with the resolution choice considering the average of the trabeculae thickness. The same patterns for the trabeculae bone should be considered for bone repair, with the new bone formation at the initial stages presenting a trabecular-like arrangement.

Other parameters during image acquisition should also be carefully defined, as the use of filters (aluminum, copper), the equipment potential and the correct location of the region that should be scanned.

Regarding to the reconstruction process, care should be taken with the correct delimitation of the region of interest, also, with the parameters such as the beam hardening correction, the smoothing and the ring artifact correction.

By the analysis of the bone parameters, the structural analysis should be able to compare different groups or conditions, following the same conditions in order to avoid bias (use of different thresholds for cortices and trabecular bone, but the same thresholds for the comparisons among the different groups). At the cortex, it should be possible to identify if one bone is more porous than the other, or if the bonne channels morphology or spatial distribution are altered in a specific condition. Within the trabecular bone, the number, thickness, size and shape of the trabeculae, together with their connectivity, should be possible to be analyzed and differentiate conditions with better or worst features related to an ideal bone quality and strength (Fig. 5).

Concerning the bone material composition, the mineral density could be evaluated and aid to differentiate health and disease condition, by means of higher or lower X-ray absorbance of the scanned bone. In this analysis, careful should be taken related to the use of a calibrated $\mu \mathrm{CT}$, by the use of phantoms of known amounts of hydroxyapatite.

Attention on the use of right nomenclatures should be taken by using guidelines and following the correct information on operator manuals of the different $\mu \mathrm{CT}$ systems.

Studies should describe all the parameters related to scanning, reconstruction, and analysis, in order to allow comparison and discussion with other studies.

Micro CT bone evaluation within dentistry has unlimited applications, including: evaluation of diseases and conditions affecting bone metabolism and structure; analysis of repair and regenerating procedures; evaluation of new therapies and surgical interventions; evaluation of the effectiveness and use of biomaterials; and more. When it is possible to analyze human jaw samples, the evaluation by this high-resolution method enables to acquire several information, both on cortices and cancellous structure and composition (Fig. 6). Both type of studies, in experimental animal conditions or human samples are able to be investigated by the use of the $\mu \mathrm{CT}$. Utilizing high-resolution
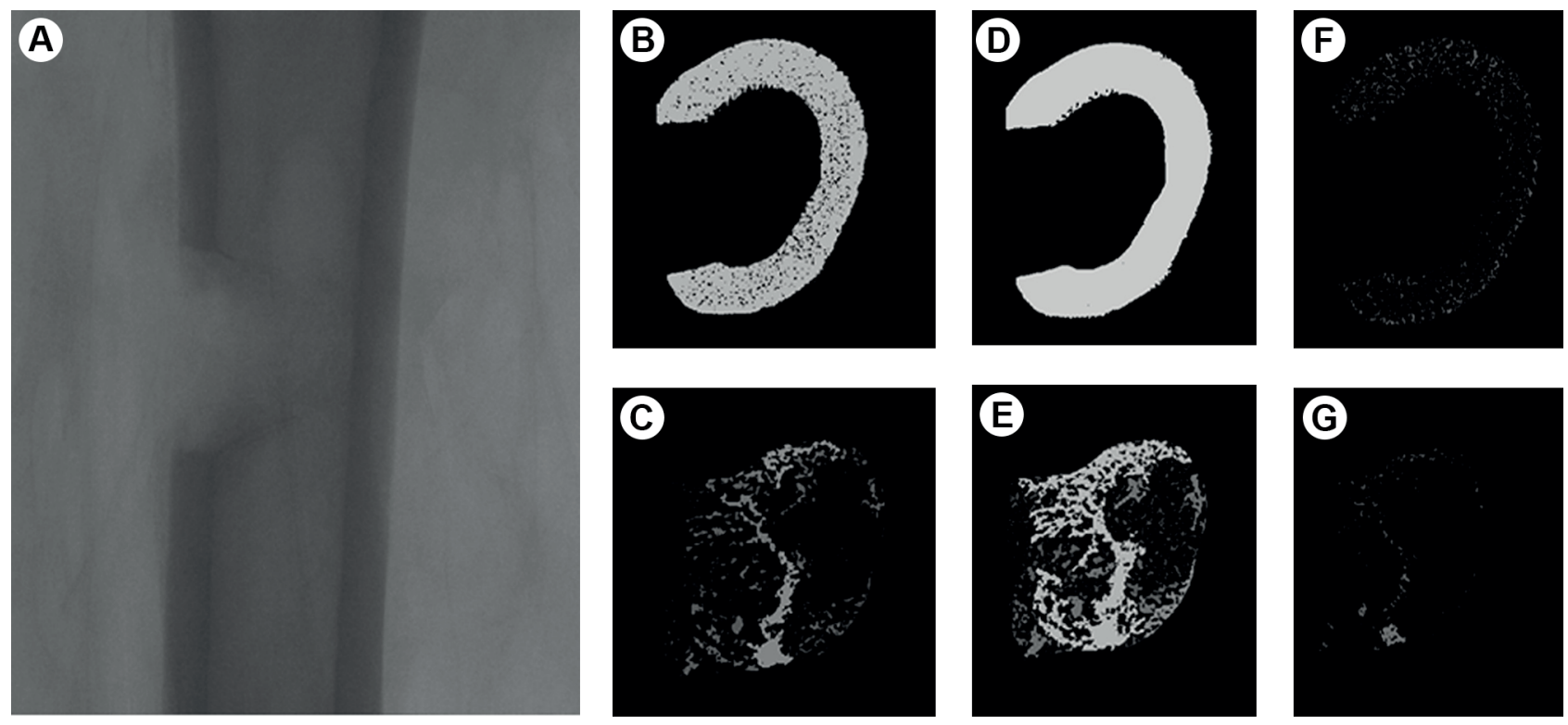

Figure 4. Rat femur with bone repair process evaluated 7 days after euthanization. A: $\mu$ CT scout view of the femur with the lesion in the middle (8 $\mu \mathrm{m}$ pixel size at $90 \mathrm{kV}, 111 \mu \mathrm{A}$ and $720 \mathrm{~ms}$ integration time; Rotation step 0.400 and Filter $\mathrm{Al} 0.5+\mathrm{Cu} 0.038$ ). B: Defined cortex after binarization with an ideal threshold. C: Defined lesion area (bone repair and medullar channel: after binarization, also with an ideal threshold. D and E: Both cortex and lesion area with over thresholding, revealing overestimation of the bone. F and G: Both cortex and lesion with under thresholding, revealing sub estimation of the bone definition. 
Choose the best resolution: go to literature to check previous information.

Calibrate the equipment for bone mineral density (using phantoms)

Check if the size of the sample allow the analysis: consider to section the sample.

Define the filter and be aware of beamhardening and ring artifact corrections.

\section{Before the scan:}

\section{Scanning process:}

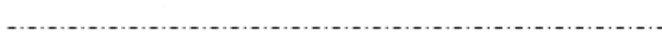

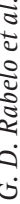

\section{Data management:}

\begin{tabular}{|c|}
\hline $\begin{array}{c}\text { Threshold: Define the threshold taking into account } \\
\text { the type, site and inherent bone characteristics. }\end{array}$ \\
\hline \hline $\begin{array}{c}\text { Consider additional measurements: Texture analysis, } \\
\text { anisotropy, fractal dimension. }\end{array}$ \\
\hline
\end{tabular}

Be aware of scan time: this will be based on the type of the sample and necessity to assess inner structures.

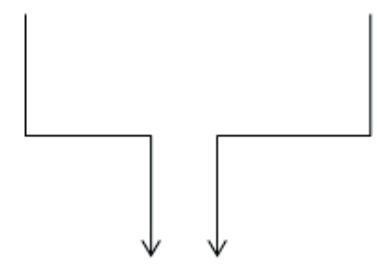
anisotropy, fractal dimension.

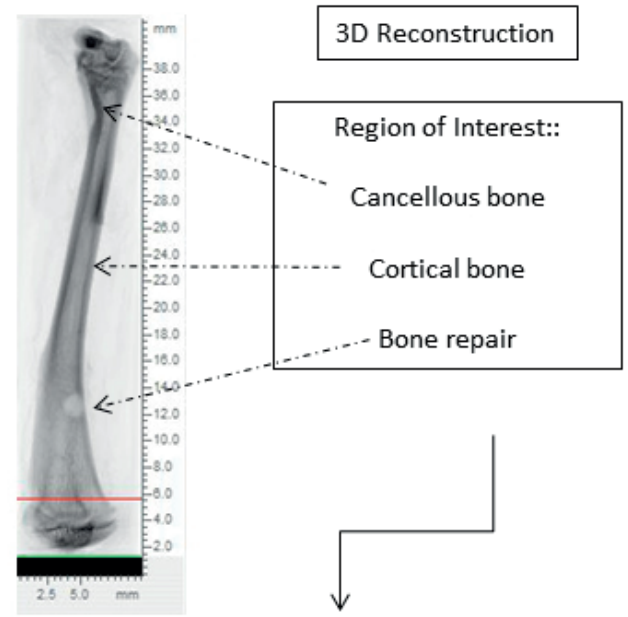

Figure 5. Flow chart with orientations for bone evaluation using microcomputed tomography. The inside figure reveals a scout view of a rat femur with a bone defect (lesion) at the diaphysis part, and both epiphysis where are possible to assess the cancellous bone.
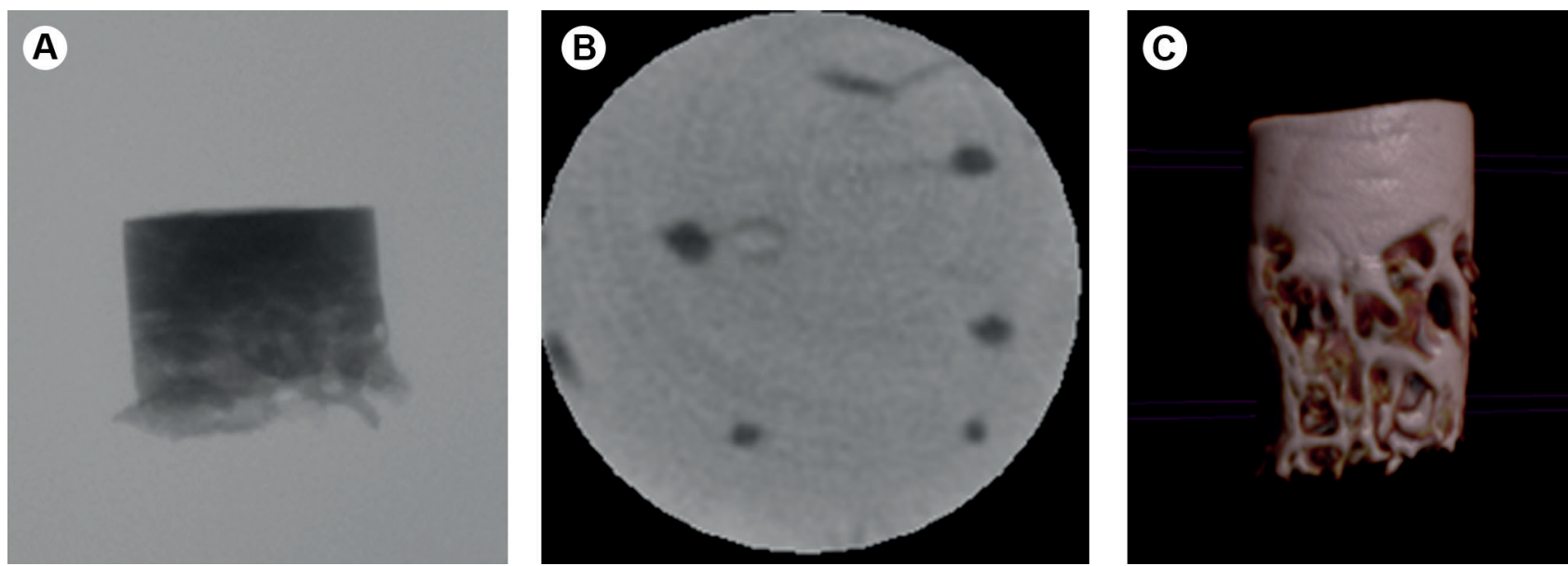

Figure 6. Human sample removed from the mandible of a patient (scan: $19 \mu \mathrm{m}$ pixel size at $50 \mathrm{kV}, 800 \mu \mathrm{A}$ and 4000 ms exposure; Rotation step 0.600 and Filter $\mathrm{Al} 0.5 \mathrm{~mm}$ ). A: Scout view revealing the bone sample, with the superior part composed by the cortex and the inferior part with cancellous bone. B: One of the slices within the cortex revealing the bone channels in dark and the bone matrix in gray. C: 3D reconstruction of the bone sample removed. 
imaging technique will allow characterization and changes in the bone tissue at the microarchitectural level.

\section{Acknowledgements}

This study was supported by the research funding agencies FAPEMIG and CAPES.

\section{Resumo}

A microtomografia computadorizada segue o mesmo princípio da tomografia computadorizada utilizada para avaliação dos pacientes, mas neste caso, é empregada para pequenas amostras com alta resolução. De forma não destrutiva, as amostras podem ser escaneadas, e cada fatia obtida é organizada de forma seriada para formar um volume tridimensional (3D). Para análise óssea, é possível obter informações de microarquitetura e composição mineral, permitindo avaliação distinta entre diferentes sitios. De acordo com as caracteristicas de cada amostra óssea, como amostras de humanos, animais, ossos longos ou achatados, epífise ou diáfise, etc, devem ser definidos os parâmetros pré-escaneamento com a resolução desejada, levando em consideração quais informações serão extraídas da avaliação. Depois do escaneamento e da reconstrução, deve-se proceder com a seleção da região de interesse (ROI), e depois seguir com o processo de binarização, que se caracteriza pela escolha de um limiar que define os voxels que compõem a região de osso e àqueles que compõem a região dos buracos (canais, áreas de reabsorção e espaço medular). No osso trabecular e no reparo os parâmetros avaliados se baseiam nas características das trabéculas e sua conectividade. No osso cortical os parâmetros estão relacionados com a espessura e porosidade. Além dos parâmetros de microarquitetura, também é possivel avaliar a densidade mineral óssea, calculada por volume de hidroxiapatita. Outros parâmetros também podem ser mensurados, utilizando técnicas computacionais como a análise de textura. Parâmetros intrínsecos e extrínsecos da qualidade óssea podem ser avaliados pela microtomografia computadorizada. Na odontologia, este método pode ser empregado em estudos que objetivem avaliar doenças, alterações metabólicas e medicamentos com repercussão no metabolismo ósseo, e na avaliação do processo de reparo e de técnicas cirúrgicas.

\section{References}

1. Metscher BD. MicroCT for comparative morphology: simple staining methods allow high-contrast 3D imaging of diverse non-mineralized animal tissues. BMC Physiol 2009;9:11.

2. Tjong W, Nirody J, Burghardt AJ, Carballido-Gamio J, Kazakia GJ. Structural analysis of cortical porosity applied to HR-p0CT data. Med Phys 2014;41:13701.

3. Bouxsein ML, Boyd SK, Christiansen BA, Guldberg RE, Jepsen KJ, Müller R. Guidelines for assessment of bone microstructure in rodents using micro-computed tomography. J Bone Miner Res 2010;25:1468-1486.

4. Burghardt AJ, Link TM, Majumdar S. High-resolution computed tomography for clinical imaging of bone microarchitecture. Clin Orthop Relat Res 2011;469:2179-2193.

5. Sinibaldi R, Conti A, Sinjari B, Spadone S, Pecci R, Palombo M, et al. Multimodal-3D imaging based on $\mu \mathrm{MRI}$ and $\mu \mathrm{CT}$ techniques bridges the gap with histology in visualization of the bone regeneration process. J Tissue Eng Regen Med 2018;12:750-761.

6. Chappard D, Baslé MF, Legrand E, Audran M. Trabecular bone microarchitecture: A review. Morphologie 2008;92:162-170.

7. Muller R, Van Campenhout H, Van Damme B, Van der Perre G, Dequeker J, Hildebrand T, et al. morphometric analysis of human bone biopsies: a quantitative structural comparison of histological sections and microcomputed tomography. Bone 1998;23:59-66.

8. Roschger $\mathrm{P}$, Paschalis EP, Fratzl $\mathrm{P}, \mathrm{Klaushofer} \mathrm{K}$. Bone mineralization density distribution in health and disease. Bone 2008;42:456-466.

9. Kohler T, Beyeler M, Webster D, Müller R. Compartmental bone morphometry in the mouse femur: Reproducibility and resolution dependence of microtomographic measurements. Calcif Tissue Int 2005;77:281-290.

10. Zebaze R, Seeman E. Cortical bone: a challenging geography. J Bone Miner Res 2015;30:24-29.

11. Farlay D, Boivin G. Bone mineral quality. Osteoporosis 2012;953-978.

12. Rocha FS, Dias PC, Limirio PHJO, Lara VC, Batista JD, Dechichi P. High doses of ionizing radiation on bone repair: is there effect outside the irradiated site? Injury 2017;48:671-673.

13. Fanuscu MI, Chang TL. Three-dimensional morphometric analysis of human cadaver bone: Microstructural data from maxilla and mandible. Clin Oral Implants Res 2004;15:213-218.

14. Rabelo GD, Coutinho-Camillo C, Kowalski LP, Portero-Muzy N, Roux J-P, Chavassieux $\mathrm{P}$, et al. Evaluation of cortical mandibular bone in patients with oral squamous cell carcinoma. Clin Oral Investig 2018;22:783-790.

15. Blok Y, Gravesteijn FA, Van Ruijven $\sqcup$, Koolstra JH. Micro-architecture and mineralization of the human alveolar bone obtained with microCT. Arch Oral Biol 2013;58:621-627.

16. Romão MMA, Marques MM, Cortes ARG, Horliana ACRT, Moreira MS, Lascala CA. Micro-computed tomography and histomorphometric analysis of human alveolar bone repair induced by laser phototherapy: A pilot study. Int J Oral Maxillofac Surg 2015;44:1521-1528.

17. Stauber M, Müller R. Micro-computed tomography: a method for the non-destructive evaluation of the three-dimensional structure of biological specimens. Methods Mol Biol 2008;455:273-292.

18. van't Hof RJ. Analysis of bone architecture in rodents using microcomputed tomography. Methods Mol Biol. Methods Mol Biol 2012;816:461-476

19. Carriero A, Doube M, Vogt M, Busse B, Zustin J, Levchuk A, et al. Altered lacunar and vascular porosity in osteogenesis imperfecta mouse bone as revealed by synchrotron tomography contributes to bone fragility. Bone 2014;61:116-124

20. Bruker-MicroCT. Bruker Micro - CT Academy Bruker Micro-CT Academy Bruker Micro-CT Acad 2015;2:1-2.

21. Kim DG, Christopherson GT, Dong XN, Fyhrie DP, Yeni YN. The effect of microcomputed tomography scanning and reconstruction voxel size on the accuracy of stereological measurements in human cancellous bone. Bone 2004;35:1375-1382.

22. Barak MM, Lieberman DE, Hublin JJ. Of mice, rats and men: Trabecular bone architecture in mammals scales to body mass with negative allometry. J Struct Biol 2013;183:123-131.

23. Christiansen BA. Effect of micro-computed tomography voxel size and segmentation method on trabecular bone microstructure measures in mice. Bone Rep 2016;5:136-140.

24. Christiansen BA. Assessment of Bone Mass and Microarchitecture in Rodents. In: Primer on the Metabolic Bone Diseases and Disorders of Mineral Metabolism: 8th Edition 2013. p. 59-68.

25. Laib a, Barou O, Vico L, Lafage-Proust MH, Alexandre C, Rügsegger P. 3D micro-computed tomography of trabecular and cortical bone architecture with application to a rat model of immobilisation osteoporosis. Med Biol Eng Comput 2000;38:326-332.

26. Shiffman D. Images and Pixels 2008. Available from: https://processing. org/tutorials/pixels/. Latest access: May 10, 2018.

27. Waarsing JH, Day JS, Weinans H. An improved segmentation method for in vivo $\mu$ CT imaging. J Bone Miner Res 2004;19:1640-1650.

28. Geusens $P$, Chapurlat $R$, Schett $G$, Ghasem-Zadeh $A$, Seeman $E_{1}$ de Jong $J$, et al. High-resolution in vivo imaging of bone and joints: a window to microarchitecture. Nat Rev Rheumatol 2014;10:304-313.

29. Bernhard A, Milovanovic P, Zimmermann EA, Hahn M, Djonic D, Krause $M$, et al. Micro-morphological properties of osteons reveal changes in cortical bone stability during aging, osteoporosis, and bisphosphonate treatment in women. Osteoporos Int 2013;24:2671-2680.

30. Gudea A, Stefan A. Histomorphometric, fractal and lacunarity comparative analysis of sheep (Ovis aries), goat (Capra hircus) and roe deer (Capreollus capreollus) compact bone samples. Folia Morphol 2013;72:13.

31. De Melo RHC, Conci A. How Succolarity could be used as another fractal measure in image analysis. Telecommun Syst 2013;52:1643-1655.

32. Russell NA, Pelletier MH, Bruce WJ, Walsh WR. The effect of gamma irradiation on the anisotropy of bovine cortical bone. Med Eng Phys 
2012;34:1117-1122.

33. Sanchez-Molina D, Velazquez-Ameijide J, Quintana V, ArreguiDalmases C, Crandall JR, Subit D, et al. Fractal dimension and mechanical properties of human cortical bone. Med Eng Phys 2013;35:576-582.

34. Fazzalari NL, Parkinson IH. Fractal dimension and architecture of trabecular bone. J Pathol. 1996;178:100-105.

35. Pothuaud L, Benhamou CL, Porion P, Lespessailles E, Harba R, Levitz P. Fractal dimension of trabecular bone projection texture is related to three-dimensional microarchitecture. J Bone Miner Res 2000;15:691699.

36. Prouteau S, Ducher G, Nanyan P, Lemineur G, Benhamou L, Courteix D. Fractal analysis of bone texture: A screening tool for stress fracture risk? Eur J Clin Invest 2004;34:137-142.

37. Dougherty G, Henebry GM. Lacunarity analysis of spatial pattern in CT images of vertebral trabecular bone for assessing osteoporosis. Med Eng Phys 2002;24:129-138.

38. Melo RHC, Vieira ED, Conci A. Characterizing the lacunarity of objects and image sets and its use as a technique for the analysis of textural patterns. Adv Concepts Intell Vis Syst Proc 2006;4179:208-219.

39. Boutroy S, Vilayphiou N, Roux JP, Delmas PD, Blain H, Chapurlat RD, et al. Comparison of 2D and 3D bone microarchitecture evaluation at the femoral neck, among postmenopausal women with hip fracture or hip osteoarthritis. Bone 2011;49:1055-1061.
40. Goldring SR, Goldring MB. Changes in the osteochondral unit during osteoarthritis: structure, function and cartilage-bone crosstalk. Nat Rev Rheumatol 2016;12:632-644.

41. Heilmeier U, Patsch JM. Diabetes and bone. Semin Musculoskelet Radiol 2016;20:300-304.

42. Marques IDB, Araújo MJCLN, Graciolli FG, Reis LM do., Pereira RM, Custódio MR, et al. Biopsy vs. peripheral computed tomography to assess bone disease in CKD patients on dialysis: differences and similarities. Osteoporos Int 2017;28:1675-1683.

43. Von Rechenberg B. Animal models in bone repair. Drug Discov Today Dis Model. Elsevier Ltd; 2014;13:23-7. Available from: http://dx.doi. org/10.1016/j.ddmod.2015.02.001.

44. Peric M, Dumic-Cule I, Grcevic D, Matijasic M, Verbanac D, Paul R, et al. The rational use of animal models in the evaluation of novel bone regenerative therapies. Bone 2015;70:73-86.

45. Freeman TA, Patel P, Parvizi J, Antoci V, Shapiro IM. Micro-CT analysis with multiple thresholds allows detection of bone formation and resorption during ultrasound-treated fracture healing. J Orthop Res 2009;27:673-679.

Received October 30, 2017

Accepted March 27, 2018 